\title{
Regulating Orthography-Phonology Relationship for English to Thai Transliteration
}

\author{
Binh Minh Nguyen \\ National University of \\ Singapore, Singapore \\ nguyen.binh.minh92eu.nus.edu
}

\author{
Gia H. Ngo \\ National University of \\ Singapore, Singapore \\ ngohgiadu.nus.edu
}

\author{
Nancy F. Chen \\ Institute for Infocomm \\ Research, Singapore \\ nfychen@i2r.a-star.edu.sg
}

\begin{abstract}
In this paper, we discuss our endeavors for the Named Entities Workshop (NEWS) 2016 transliteration shared task, where we focus on English to Thai transliteration. The alignment between Thai orthography and phonology is not always monotonous, but few transliteration systems take this into account. In our proposed system, we exploit phonological knowledge to resolve problematic instances where the monotonous alignment assumption breaks down. We achieve a $29 \%$ relative improvement over the baseline system for the NEWS 2016 transliteration shared task.
\end{abstract}

\section{Introduction}

Transliteration is the process of transforming a word from one writing system (source word) to a word in another writing system (target word) (Knight and Graehl, 1998). Transliteration is often used to borrow names and technical terms from the source language into the target language when translation is difficult or awkward. For example, British is transliterated into Thai as [ bri-tit ] using Royal Thai General System of Transcription (RTGS) notation (Royal Institute, 1999).

Transliteration can be formulated as a special case of translation. Instead of converting words from one language to the semantically equivalent words in another language, transliteration converts the source word to a phonetically equivalent target word (Knight and Graehl, 1998).

In transliteration, character-reordering is important to ensure the transliterated words follow the phonotactic rules of the target language. Character-reordering in transliteration is similar to word-reordering in translation, where the translated sentence needs to satisfy the grammatical rules of the target language. However, most transliteration systems do not take into account character-reordering.

If the phonetically equivalent characters are reordered in the target word, the source word and the transliterated word are said to be nonmonotonously aligned (Toms and Casacuberta, 2006). A classical approach to transliteration is using phrase-based Statistical Machine Translation (pbSMT). While pbSMT approach can model non-monotonous alignment of characters in the transliteration task, the pbSMT systems introduced in Kunchukuttan and Bhattacharyya (2015), Nicolai et al. (2015) and Finch et al. (2015) did not model such character-reordering. In addition to pbSMT, Nicolai et al. (2015) also used graphemeto-phoneme (G2P) conversion tools, namely DirecTL+ (Jiampojamarn et al., 2010) and Sequitur G2P (Bisani and Ney, 2008), for the transliteration task. Such G2P conversion tools also make a similar assumption of monotonous alignment between source and target word. While this assumption is reasonable for transliteration between most language pairs, there are cases in English to Thai transliteration whereby this assumption is invalid.

In this paper, we regulate the relationship between Thai orthography and phonology in the English to Thai transliteration task. We show that the transliteration accuracy can be improved by addressing the mismatch between Thai orthography and phonology that causes the monotonous alignment assumption to break down.

\section{Thai Phonology}

\subsection{Syllable}

A syllable is considered the basic unit of a word in both written and spoken language (Ladefoged and Johnson, 2014). Most languages have the following syllable structure, including English and Thai (Kessler and Treiman, 1997; Luksaneeyanawin, 1992): 


\section{$[O] \quad N \quad[C d] \quad[T]$}

where $O, N, C d, T$ denotes onset, nucleus, coda, tone respectively.

In Thai, an onset $(O)$ has at most two consonants and a coda $(C d)$ has at most one consonant, while a nucleus $(N)$ can be a vowel or a diphthong (Luksaneeyanawin, 1992). Tone $(T)$ is a feature of many tonal languages (Yip, 2002). Tone is a variation in pitch that is used to distinguish different words (Yip, 2002). For example, both the Thai words value and to trade have the same syllable [ khaa ] (RTGS), but value is pronounced with a falling tone while to trade is pronounced with a high tone.

The aim of transliteration is to generate a word in the target language that best matches the pronunciation of the word in the source language (Knight and Graehl, 1998). Although the syllable structure in English and in Thai are similar, the idiosyncratic relationship between Thai pronunciation and Thai orthography makes English to Thai transliteration complex.

1. In Thai orthography, the position of the onset and nucleus can be inverted for a syllable with a leading vowel (Chotimongkol and Black, 2000). A leading vowel is part of the syllable's nucleus. While the vowels of the nucleus are pronounced after the consonants of the onset as specified by the syllable structure in (1), leading vowels precede the consonants of the onset in written form. The order of consonants and vowels in Thai written form therefore does not always match the pronunciation order. This is unlike English whereby the order of consonants and vowels in the written form matches the order in pronunciation. For example, Reagan is transliterated into Thai as [er-aekn ] (RTGS) but is pronounced in Thai as [re:ke:n] (IPA). [ $e$ ] and $[e a]$ are leading vowels and are written before their corresponding onset which are [ $r$ ] and [ $k$ ] respectively.

2. Lexical tones in Thai are not uniquely defined by tone marks, but are determined by the type of the syllable, the class of the onset consonant, and the length of the nucleus (Smyth, 2002). For example, the Thai words for both to pass and glancing are pronounced with a low tone. However, only to pass has a tone mark in Thai script, while glancing does not.

3. Some vowels are implicit in certain phonetic contexts (Chotimongkol and Black, 2000). Such vowels are present when the syllable is pronounced but it is omitted when the syllable is transcribed. For example, Steve is transliterated as [s-tip ] but is pronounced as [ sa-tip ] (RTGS). In this case, the vowel [ $a$ ] is implicit.

In this work, we focus on the onset-nucleus inversion case (case 1), which occurs much more often.

\subsection{Alignment between Thai Orthography and Phonology}

Even though the syllable structure of English and Thai are similar, the mismatch between Thai orthography and Thai syllable structure can lead to challenges in aligning English-Thai transliteration word pairs. In this section, we describe how onsetnucleus inversion may make monotonous alignment difficult.

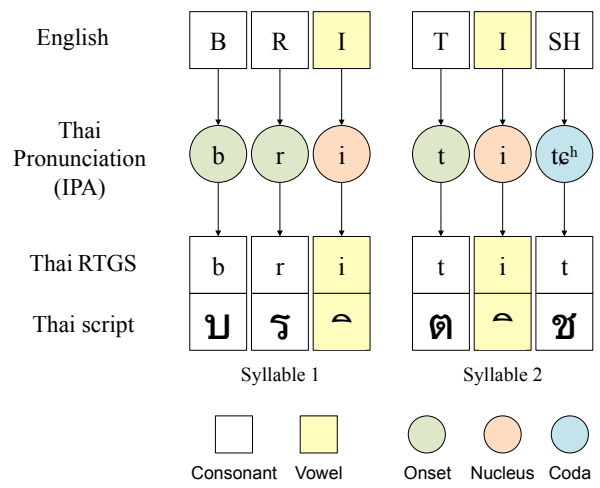

Figure 1: Monotonous Alignment, the source word is British

When there is no onset-nucleus inversion such as the case in Figure 1, the alignment between the English word and the Thai word is monotonous.

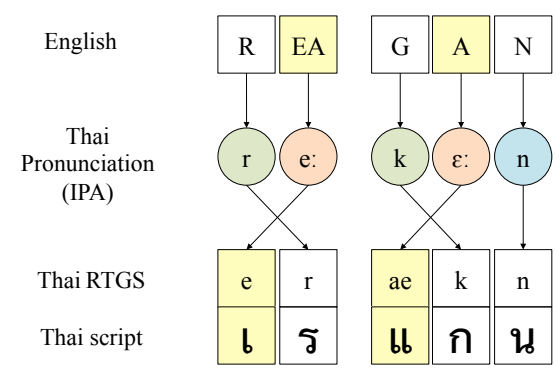

Figure 2: Non-Monotonous alignment of EnglishThai transliteration pairs due to onset-nucleus inversion of monophthongs.

However, when onset-nucleus inversion occurs such as the case in Figure 2, the alignment is 
non-monotonous. Under the monotonous alignment assumption, the English onset [ R ] may be wrongly aligned to the Thai nucleus $[e]$, and the English nucleus [ EA ] may be wrongly aligned to the Thai onset $[r]$. The English syllable [ REA ] may still be aligned correctly to the Thai syllable [ er ] if the syllables appear together frequently in the training data. Nevertheless, the presence of onset-nucleus inversion increases the number of possible alignments between EnglishThai transliteration pairs.

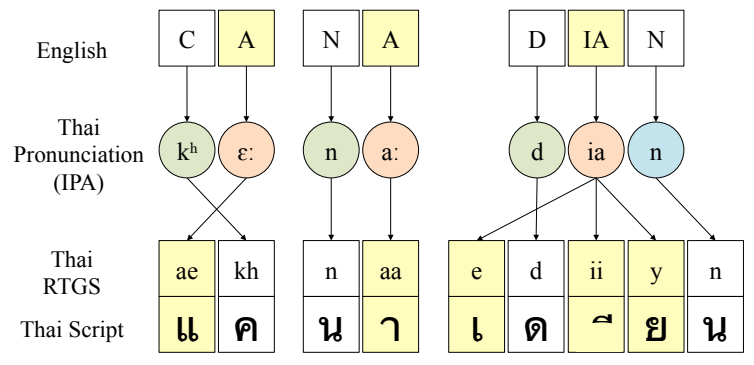

Figure 3: Non-Monotonous alignment of EnglishThai transliteration pairs due to onset-nucleus inversion of diphthongs.

Onset-nucleus inversion also occurs in syllables with diphthongs. In Figure 3, the nucleus of the third syllable, namely $[e][i i][y]$, is a diphthong in Thai, which comprises of three phonemes $[e],[i i]$ and $[y]$. Although $[e],[i i]$ and [ $y$ ] are components of the same nucleus, they are not adjacent. Under the monotonous alignment assumption, it is unclear how to align the English onset, nucleus and coda with Thai onset, nucleus and coda respectively. We attempt to address these issues in the proposed transliteration system.

\section{Baseline Systems}

We considered two classic approaches as our baseline systems. Transliteration seeks to convert an English string $\mathbf{f}=\left(f_{1}, f_{2}, \ldots, f_{n}\right)$ to a Thai string $\mathbf{e}=\left(e_{1}, e_{2}, \ldots, e_{m}\right)$.

\subsection{Phrase-based Statistical Machine Translation System}

Under the pbSMT system, the objective function is given by:

$$
\mathbf{e}^{*}=\arg \max _{\mathbf{e}} p(\mathbf{e}) p(\mathbf{f} \mid \mathbf{e}),
$$

where $p(\mathbf{e})$ is estimated from an n-gram language model of Thai, and $p(\mathbf{f} \mid \mathbf{e})$ is estimated from the alignment of segments (phrases) of $f$ with segments (phrases) of $\mathbf{e}$.

We implement the pbSMT system with the Moses toolkit (Koehn et al., 2007). We use GIZA++ (Och and Ney, 2003) to perform align- ment, and SRILM (Stolcke, 2002) to train a 5-gram language model of Thai transliteration units with Witten-Bell smoothing (Witten and Bell, 1991). While the pbSMT systems implemented in (Kunchukuttan and Bhattacharyya, 2015) and (Nicolai et al., 2015) did not model word reordering, we tried various reordering models offered by the Moses toolkit.

\subsection{Joint Source-channel System}

The baseline system is based on the joint source-channel model, formulated for transliteration in ( $\mathrm{Li}$ et al., 2004). A similar model (joint sequence model) was proposed for grapheme-tophoneme conversion in (Bisani and Ney, 2002). We use the Sequitur G2P tool from (Bisani and Ney, 2008) to train the joint source-channel model by assuming a direct correspondence between phoneme and grapheme in the target language.

Given an English string $\mathbf{f}$ and a Thai string e, the joint source-channel model estimates the cosegmentation $\mathbf{q}$, defined as $\mathbf{q}=\left(q_{1}, q_{2}, \ldots, q_{n}\right)$ where, $q_{i}=\left(f_{i}, e_{i}\right), \mathbf{f}=\left(f_{1}, f_{2}, \ldots, f_{n}\right)$ and $\mathbf{e}=\left(e_{1}, e_{2}, \ldots, e_{n}\right)$. During decoding, the output Thai string corresponds to the co-segmentation that matches the input English string and yields the maximum likelihood.

The monotonous alignment assumption is built into the joint source-channel model (Bisani and Ney, 2008). Under this assumption, $\forall i<j, f_{i}$ appears before $f_{j}$ and $e_{i}$ appears before $e_{j}$. In a Thai syllable with onset-nucleus inversion, $e_{i}$ corresponds to a leading vowel and $e_{i+1}$ corresponds to the onset that comes after the leading vowel. However, $f_{i}$ and $f_{i+1}$ may still be matched to the onset and nucleus in the English syllable. Therefore, the model may be confused, as the English onset is matched to the Thai nucleus, and the English nucleus is matched to the Thai onset.

\section{Proposed Augmented System}

The proposed system (Figure 4) augmented the joint source-channel model with a vowel-onset transposition step to regulate the syllable structure.

During training, for Thai syllables with onsetnucleus inversion, the vowel-onset transposition step swaps the location of the leading vowel and the onset consonant (see next page). This swapping ensures that the nucleus always occurs after the onset in a syllable, and vowels that belong to the same nucleus are adjacent in the Thai script.

During decoding, Thai entries that have undergone vowel-onset transposition are reverted back 


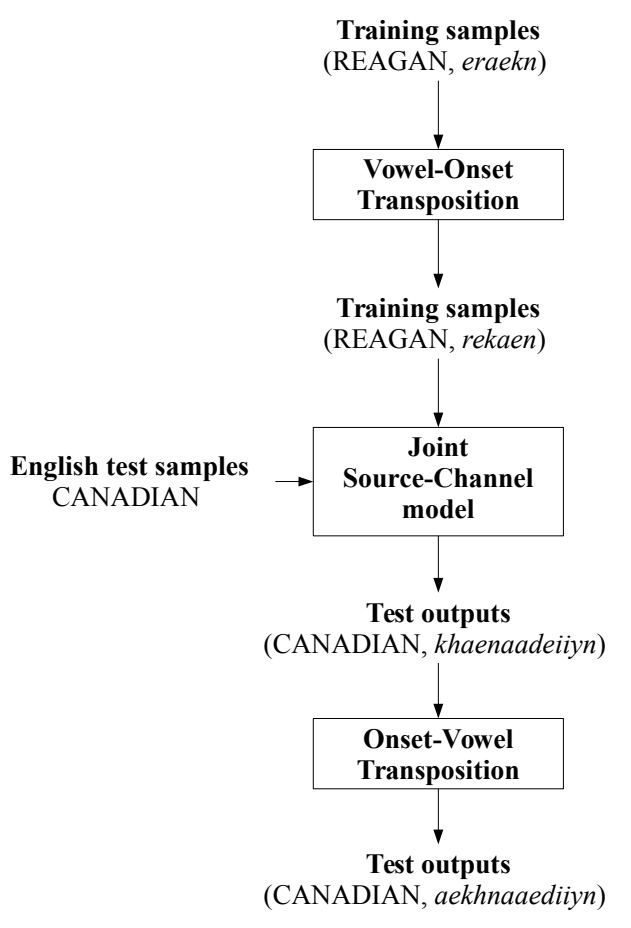

Figure 4: Augmented System. English words are capitalized, Thai words are italicized RTGS.

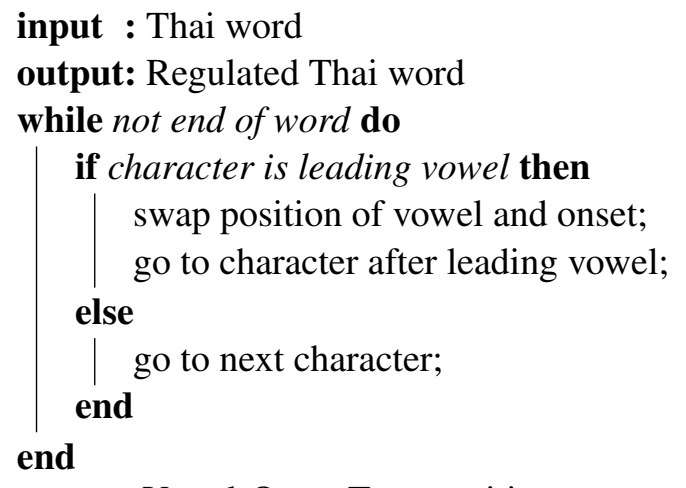

via onset-vowel transposition.

\section{Experiments}

We used the TOP-1 metric (Banchs et al., 2015) for performance comparison between the baseline and the augmented systems. As denoted in Table 1, 75\% of the NEWS2016 training set was used for training, the remaining $25 \%$ of the NEWS2016 training set was used for tuning, and the NEWS2016 dev set was used for testing. A 6-gram joint source-channel model was used for Baseline joint S-C and Augmented 1. Reordering option 'msd-bidirectional-fe' in Moses was used for the Baseline pbSMT system as it yielded the best TOP-1 metric.

From Table 2, on the NEWS2016 test set, the

\begin{tabular}{|l|l|}
\hline System & Set-up \\
\hline Baseline pbSMT & Train = 75\% NEWS 2016 training \\
Baseline joint S-C & Tune = 25\% NEWS 2016 training \\
Augmented 1 & \\
\hline Augmented 2 & $\begin{array}{l}\text { Train = NEWS 2016 training } \\
\text { Tune = NEWS 2016 dev }\end{array}$ \\
\hline
\end{tabular}

Table 1: Data partitioning for the different systems. (pbSMT: phrase-based statistical machine translation, joint $\mathrm{S}-\mathrm{C}$ : joint source-channel)

\begin{tabular}{|l|c|c|}
\hline System & Dev Set & Test Set \\
\hline Baseline pbSMT & 0.3117 & 0.111650 \\
Baseline joint S-C & 0.3662 & 0.117314 \\
Augmented 1 & 0.4015 & 0.144013 \\
Augmented 2 & NA & 0.155340 \\
\hline
\end{tabular}

Table 2: Results on NEWS 2016 shared task in terms of TOP-1 accuracy.

augmented system (Augmented 1) achieves a 29\% relative improvement over the pbSMT baseline system (Baseline pbSMT), and a $23 \%$ relative improvement over the joint source-channel baseline system (Baseline joint S-C).

To observe the performance of the augmented system with the full training data, we trained another separated augmented system (Augmented 2 ), using both the training set and the dev set data as denoted in Table 1. A 6-gram joint sourcechannel model was also used for this augmented system. Despite a simpler setup due to exploiting phonology knowledge, this system achieves comparable performance to that of systems reported in (Nicolai et al., 2015) and (Finch et al., 2015) for English to Thai transliteration task.

\section{Discussion}

Besides the augmented system, we explored a rule-based approach and a phonology-augmented statistical approach for the English to Thai transliteration task. Phonology-augmented statistical approach for English to Vietnamese transliteration has been proposed in (Ngo et al., 2015). These two approaches have inspired the vowel-onset transposition strategy for the joint source-channel model. Despite being similar, our proposed approach takes into account the complex relationship between phonology and orthography, which was not considered in (Ngo et al., 2015). We are working on generalizing this relationship into a statistical model, and also to address other peculiar characteristics of Thai script. 


\section{References}

Rafael E. Banchs, Min Zhang, Xiangyu Duan, Haizhou Li, and A. Kumaran. 2015. Report of NEWS 2015 Machine Transliteration Shared Task. In Proceedings of NEWS 2015 The Fifth Named Entities Workshop, page 10 .

Maximilian Bisani and Hermann Ney. 2002. Investigations on joint-multigram models for grapheme-tophoneme conversion. In INTERSPEECH.

Maximilian Bisani and Hermann Ney. 2008. Jointsequence models for grapheme-to-phoneme conversion. Speech Communication, 50(5):434-451.

Ananlada Chotimongkol and Alan W. Black. 2000. Statistically trained orthographic to sound models for Thai. In INTERSPEECH, pages 551-554.

Andrew Finch, Lemao Liu, Xiaolin Wang, and Eiichiro Sumita. 2015. Neural Network Transduction Models in Transliteration Generation. In Proceedings of NEWS 2015 The Fifth Named Entities Workshop, page 61 .

Sittichai Jiampojamarn, Colin Cherry, and Grzegorz Kondrak. 2010. Integrating joint n-gram features into a discriminative training framework.

Brett Kessler and Rebecca Treiman. 1997. Syllable structure and the distribution of phonemes in english syllables. Journal of Memory and Language, 37(3):295-311.

Kevin Knight and Jonathan Graehl. 1998. Machine transliteration. Computational Linguistics, 24(4):599-612.

Philipp Koehn, Hieu Hoang, Alexandra Birch, Chris Callison-Burch, Marcello Federico, Nicola Bertoldi, Brooke Cowan, Wade Shen, Christine Moran, Richard Zens, et al. 2007. Moses: Open source toolkit for statistical machine translation. In Proceedings of the 45th annual meeting of the ACL on interactive poster and demonstration sessions, pages 177-180. Association for Computational Linguistics.

Anoop Kunchukuttan and Pushpak Bhattacharyya. 2015. Data representation methods and use of mined corpora for Indian language transliteration. In Proceedings of NEWS 2015 The Fifth Named Entities Workshop, page 78 .

Peter Ladefoged and Keith Johnson. 2014. A course in phonetics. Cengage learning.

Haizhou Li, Min Zhang, and Jian Su. 2004. A joint source-channel model for machine transliteration. In Proceedings of the 42nd Annual Meeting on association for Computational Linguistics, page 159. Association for Computational Linguistics.

Sudaporn Luksaneeyanawin. $1992 . \quad$ Threedimensional phonology: a historical implication. In Proceedings of the Third International Symposium on Language and Linguistics: Pan Asiatic Linguistics, volume 1, pages 75-90.

Hoang Gia Ngo, Nancy F. Chen, Binh Minh Nguyen, Bin Ma, and Haizhou Li. 2015. PhonologyAugmented Statistical Transliteration for LowResource Languages. In Sixteenth Annual Conference of the International Speech Communication Association.

Garrett Nicolai, Bradley Hauer, Mohammad Salameh, Adam St Arnaud, Ying Xu, Lei Yao, and Grzegorz Kondrak. 2015. Multiple System Combination for Transliteration. In Proceedings of NEWS 2015 The Fifth Named Entities Workshop, page 72.

Franz Josef Och and Hermann Ney. 2003. A systematic comparison of various statistical alignment models. Computational linguistics, 29(1):19-51.

Royal Institute. 1999. Principles of romanization for thai script by transcription method.

D. Smyth. 2002. Thai: An Essential Grammar. Essential grammar. Routledge.

Andreas Stolcke. 2002. Srilm-an extensible language modeling toolkit. In INTERSPEECH, volume 2002, page 2002.

Jess Toms and Francisco Casacuberta. 2006. Statistical phrase-based models for interactive computerassisted translation. In Proceedings of the COLING/ACL on Main conference poster sessions, pages 835-841. Association for Computational Linguistics.

Ian H Witten and Timothy C Bell. 1991. The zerofrequency problem: Estimating the probabilities of novel events in adaptive text compression. Information Theory, IEEE Transactions on, 37(4):10851094.

Moira Yip. 2002. Tone. Cambridge University Press. 